DOI: http://dx.doi.org/10.18569/tempus.v11i1.1922 /

\title{
GTTrans: estratégia de aproximação dos múltiplos saberes sobre a saúde integral das pessoas trans em uma Universidade Pública
}

\section{GTtrans: múltiple estrategia de abordaje de conocimientos sobre la salud general de las personas trans en una universidad pública}

\section{GTTrans: multiple knowledge approach strategy on the overall health of trans people in a public university}

Jose Humberto Guimarães Santos ${ }^{1}$

Kelly Silva

Priscilla Daiana Figueiredo Souza ${ }^{3}$

Rodrigo Dornelas ${ }^{4}$

RESUMO: A transexualidade é ainda entendida como transtorno de personalidade pelos sistemas classificatórios de doenças, especialmente pela associação americana de psiquiatria. Com o intuito de refletir sobre essa classificação, assim como apresentar questões de cuidados em saúde à pessoas trans, surgiu o grupo intitulado "GTTrans: Transexualidade em Pauta" sobre saúde da pessoa trans dentro do Projeto de Extensão da Universidade Federal de Sergipe, Campus Prof. Antonio Garcia Filho, na cidade de Lagarto. Aberto para toda a comunidade interna e externa à universidade, propôs-se que os encontros seriam uma oportunidade $\mathrm{d} @ \mathrm{~s}^{5}$ estudantes e comunidade em geral de conhecer as necessidades específicas e gerais das pessoas trans nos serviços de saúde, propondo melhorias na rede de atenção envolvida com a universidade.

Palavras-chave: Transexualidade; Acesso aos Serviços de Saúde; Necessidades e Demandas nos Serviços de Saúde.

RESUMEN: La transexualidad es todavía entendida como trastorno de personalidad por los sistemas clasificatorios de enfermedades, especialmente por la asociación americana de psiquiatría.

1 Graduando em Medicina pela Universidade Federal de Sergipe - Campus Prof Antonio Garcia Filho. Email: josehumbertoguimaraes@gmail.com.

2 Universidade Federal de Sergipe - Campus Prof Antonio Garcia Filho. Email: kelly.fonoufs@gmail.com

3 Universidade Federal de Sergipe - Campus Prof Antonio Garcia Filho. Email: lanadoza@yahoo.com.br

4 Universidade Federal de Sergipe - Campus Prof Antonio Garcia Filho. Email: odrigodornela@uol.com.br

5 O símbolo “@” foi colocado em todas as palavras que segregariam por gênero, uma vez que o que motiva o trabalho é a igualdade de gêneros, sem privilégios ou direcionamentos. 
Con el fin de discutir sobre esa clasificación, así como presentar cuestiones de atención en salud a las personas trans, surgió el grupo continuado "GTTrans: Transexualidad en Pauta" sobre salud de la persona trans dentro del Proyecto de Extensión de la Universidad Federal de Sergipe, Campus Prof. . Antonio García Filho, en la ciudad de Lagarto. Abierto para toda la comunidad interna y externa a la universidad, se propuso que los encuentros serían una oportunidad de los estudiantes y la comunidad en general de conocer las necesidades específicas y generales de las personas trans en los servicios de salud, proponiendo mejoras en la red de salud Atención a la universidad.

Palabras clave: Transexualidad; El acceso a los Servicios de Salud; Necesidades y demanda de servicios sanitarios.

ABSTRACT: Transsexuality is still understood as personality disorder by disease classificatory systems, especially by the American Association of Psychiatry. In order to reflect about this classification and to present questions of care in health to trans people emerged the group entitled "GTTrans: Transexualidade em Pauta" about trans person health within the Extension Project of the Federal University of Sergipe, Campus Prof. Antonio Garcia Filho, in the city of Lagarto. Open to all internal and external community, it was proposed that the meetings would be an opportunity for students and the community in general to meet the specific and general needs of transgender people in health services, proposing improvements in the network of attention Involved with the university.

Keywords: Transsexuality; Access to Health Services; Needs and demands on health services.

\section{INTRODUÇÃO}

A portaria $\mathrm{n}^{\mathrm{o}} 2.836^{1}$ de 2011, institui a Política Nacional de Saúde Integral de Lésbicas, Gays, Bissexuais, Travestis e Transexuais (LGBT) tem como diretriz o respeito aos direitos humanos das pessoas LGBT, contribuindo para a eliminação do estigma e da discriminação decorrentes das homofobias, lesbofobias, gayfobias, bifobias, travestifobias e transfobias, consideradas determinantes sociais de saúde, por influenciar na qualidade de vida das pessoas, diretamente na ocorrência de problemas de saúde.

O Processo Transexualizador realizado pelo SUS, instituído pelas Portarias $n^{\circ} 1.707^{2}, n^{\circ} 457^{3}$ de agosto de 2008 e ampliado pela Portaria $\mathrm{n}^{\mathrm{o}} 2.803^{4}$, de 19 de novembro de 2013, visa garantir o atendimento integral de saúde às pessoas trans. Porém ainda se observam elementos, dentre eles, o preconceito, que dificultam o atendimento, associado ao despreparo dos profissionais de saúde. Tais características podem ser justificadas pela ausência de espaços acadêmicos que discutam gênero e, especificamente, os cuidados em saúde necessários a essa população.

Deste modo, para que se pudesse minimizar a lacuna na formação desses profissionais relacionadas à saúde de pessoas trans, foi criado o grupo de estudos "GTTrans: Transexualidade em pauta”. O objetivo do grupo é capacitar discentes e população local interessada na temática sobre 
as necessidades em saúde de pessoas trans. Foram promovidas ações educativas direcionadas aos profissionais da área de saúde, professor@s e estudantes.

Os encontros acontecem mensalmente, com duração média de duas horas por dia e as discussões e reflexões propostas tem como fio condutor a Política Nacional de Saúde Integral LGBT, privilegiando os seguintes aspectos: discussão sobre conceitos relacionados a identidade de gênero: transgeneralidades (transexualidade/travestilidade) e funcionalidade (Crossdressers ,Drag queen/king etransformista); estratégias em promoção de melhorias no acesso de pessoas trans aos serviços públicos de saúde; capacitação d@s profissionais de saúde, professor@s e estudantes a lidarem com as necessidades específicas de pessoas trans no acesso aos serviços de saúde.

O presente relato de experiência tem como objetivo apresentar as discussões desenvolvidas no GTTrans, para que assim possa subsidiar outros espaços e instituições de ensino sobre as necessidades específicas da população trans; e ainda divulgar o mecanismo utilizado na tentativa de extinguir o preconceito e seu impacto negativo no atendimento à saúde dessas pessoas.

\section{DESENVOLVIMENTO}

O "GTTrans: Transexualidade em pauta" surgiu como proposta de intervenção nas graduações de saúde do Campus Universitário "Prof. Antônio Garcia Filho" da Universidade Federal de Sergipe - UFS -, na cidade de Lagarto. Dentro dos projetos pedagógicos dos cursos pode-se observar que pouco, ou nada, se discute sobre questões de gênero e seus impactos nas determinações sociais de saúde. O campus tem um perfil inovador, com metodologias ativas em todos os oito cursos implantados - enfermagem, farmácia, fisioterapia, fonoaudiologia, medicina, nutrição, odontologia, e terapia ocupacional. O espaço de formação dos profissionais da área de saúde deve oportunizar discussões sobre a saúde integral de todas e todos, sem distinção, quaisquer que sejam. Nesta perspectiva percebeu-se que o GTTrans poderia contribuir com a lacuna dos projetos políticos que não contemplam esta discussão e assim aproximar discentes e docentes da temática de modo que pudessem se apropriar e tornarem-se multiplicadores das necessidades em saúde desta população.

A realização do I Fórum de Saúde Integral Trans na cidade de Lagarto, promovido pelo Departamento de Fonoaudiologia no fim de 2015, foi o início das discussões do tema no referido campus. Os dois dias do evento tiveram participações de pesquisadores, ativistas e profissionais da área da saúde que explicaram sobre as necessidades específicas e as estratégias terapêuticas aplicadas às pessoas trans. No ano seguinte projetos de pesquisa e extensão tiveram como enfoque a saúde dessa população e possibilitaram discussões em espaços permanentes durante o ano letivo que puderam se aprofundar em conceitos e termos sobre o tema.

Assim, propôs-se o GTTrans dentro do projeto de extensão "Transexualidade e voz: estratégias para saúde vocal", vinculado à criação de um ambulatório fonoaudiológico para pessoas trans na Clínica-Escola de Fonoaudiologia da UFS no Campus de Lagarto. Com a inserção de discentes dos 
cursos de medicina e enfermagem no projeto, surgiu a necessidade de se ampliar a discussão e de aprofundamento do tema, que interfere em suas práticas clínicas, melhorando-os como cidad@s, mas principalmente como profissionais. Deste modo, ao ampliar a abordagem multidisciplinar no atendimento em saúde à pessoa trans, o projeto se caracterizou como uma estratégia para efetivar as propostas de discutir gênero, reconhecer os direitos já estabelecidos das pessoas trans nos serviços de saúde, problematizar a atuação dos profissionais de saúde diante da transexualidade, além de abrir a universidade para essas pessoas ao convidá-las para a construção e participação nos projetos.

Os encontros tiveram temáticas que se relacionavam com as discussões científicas publicadas nos últimos anos, conforme pesquisa bibliográfica realizada pel@s participantes no período de planejamento. Embora preestabelecidos, os temas foram flexibilizados de acordo com as necessidades dos movimentos sociais com os quais o projeto manteve contato, pelo contexto político regional e nacional e com as sugestões dos participantes.

Entre fevereiro e março de 2016, foi realizado o cadastro do GTTrans no Sistema Integrado de Gerenciamento e Administração Acadêmica - SIGAA, portal que reúne todas as atividades da UFS; e assim deu-se início ao processo de divulgação e período de inscrições para participação. Inicialmente, oferecemos 30 vagas, que se esgotaram, fazendo com que ampliássemos a quantidade de inscritos. Ao final, obteve-se 66 inscritos, graduandos dos cursos do referido campus, além de estudantes oriundos de outros campi da universidade. Também tivemos inscritos de universidades particulares da Bahia e Sergipe, além de representantes de movimentos sociais de diversas localidades.

No mês de março de 2016 a discussão foi em torno de Gênero e Identidade de Gênero. Nesse encontro participaram uma representante da AmoSerTrans - Associação e Movimento Sergipano de Transexuais e Travestis - e uma da ASTRAL - Associação de Travestis de Lagarto. Para pontapé da discussão foi utilizado o documentário "De gravata e unha vermelha", disponível para locação em plataforma digital. O documentário traz depoimentos de diversas personalidades brasileiras que transitam entre os gêneros. Neste documentário questiona-se o ser-homem ou ser-mulher. Nesta perspectiva, há um estudo ${ }^{6}$ que discute como potencial transformador de conceitos usados nos estudos mais recentes no campo do gênero e da sexualidade e é fortalecida pelos exemplos que o documentário apresenta de pessoas públicas como a cartunista Laerte, a cantora Mel Gonçalves e o cantor Ney Matogrosso, que mostraram as diversas possibilidades que há para identificação do indivíduo enquanto ser social. A presença das duas representantes dos movimentos sociais pode despertar uma curiosidade produtiva n@s participantes: diversas perguntas sobre a forma como encaram as questões de gênero e as discriminações surgiram, sendo enriquecedora para o direcionamento do debate.

O segundo encontro ocorreu em abril de 2016 e teve a participação de uma representante da AmoSerTrans (Associação e Movimento Sergipano de Travestis e Transexuais). Nele pode- 
se entender como o termo travesti foi sendo incorporado ao movimento GLS - que passou a ser chamado LGBT; foi apresentada a história do movimento Trans em Sergipe e sua importância no cenário nacional. Em uma publicação pode-se ter o percurso histórico de construção das identidades coletivas e a organização dos movimentos representativos ${ }^{7}$. A Unidas - Associação das Travestis na Luta pela Cidadania - foi a primeira organização sergipana e a quinta nacional ${ }^{8}$. O Estado de Sergipe tem um histórico de lutas por direitos e visibilidade que impulsionou as conquistas já alcançadas. A politização das travestis e transexuais do estado encorajaram a participação na política partidária, exemplo de Linda Brasil, candidata às eleições na cidade de Aracaju nas eleições de 2016.

As necessidades em saúde também foram explicitadas nesse encontro. Percebeu-se que a possibilidade de um ambulatório Trans no Estado tem um efeito positivo na esperança das meninas e meninos Trans de terem seus corpos adequados à sua identidade de gênero. Ainda foi apontada a necessidade urgente de que todo e qualquer serviço de saúde coloque em prática a Política Nacional de Saúde LGBT, que, entre outras condutas, garante a utilização do nome social para as pessoas trans.

No terceiro encontro não houve participação de nenhum@ representante de movimentos, por questões logísticas. O tema abordado foi "O processo de acolhimento de pessoas trans nos serviços de saúde", e a discussão desencadeada pel@s participantes do GTTrans mostrou como@s inscrit@s estavam atent@s ao debate de gênero e seu impacto nos serviços de saúde. Discutiu-se sobre as dificuldades que@s profissionais de saúde têm em realizar os atendimentos direcionados às pessoas trans, que pode ser justificado pela ausência de contato com essa população, por motivos religiosos ou formação sociocultural, o que precariza o serviço baseado nos princípios de universalidade e equidade do SUS $^{9}$. Neste caso refletiu-se sobre como um profissional preparado pode contribuir positivamente nos cenários de prática e trabalho.

Em junho a discussão abordou a educação da pessoa trans. Com presença de militantes da ASTRAL e AmoSerTrans, percebeu-se como as meninas e meninos trans são discriminad@s na escola, sendo impedid@s de estarem naquele ambiente que, por conta do preconceito generalizado, se torna opressor e hostil para quem não se adequa aos padrões impostos socialmente. Essa heteronormatização do ambiente escolar pode ser apresentada como excludente e discriminatória de quem não se adequa à convenção social de se envolver sexualmente com o sexo oposto, quando adicionado à visão de negação do gênero ao qual sua genitália foi relacionada em seu nascimento, se torna um mecanismo cruel de sofrimento ao impedir a livre manifestação das identidades trans ${ }^{9}$. A questão educacional foi tratada por ser um determinante social de saúde, ao mesmo tempo em que envolve globalmente as questões da transexualidade.

"SUS na prática: vivência nos Serviços de Saúde" foi tema do encontro de julho. Nele, além da militante, mestranda em Educação, assistente social e professora lotada na Secretaria Municipal de Educação da cidade de Lagarto, a sergipana Adriana Lohanna, contou-se com a participação, por vídeo-conferência, da pesquisadora, assistente social da Secretaria de Saúde do Distrito Federal 
Adelyany Batista e de Sérgio Araújo, um dos fundadores do ambulatório Trans da Paraíba. Tod@s @s participant@s puderam expor as dificuldades que as pessoas trans têm ao tentarem acesso aos serviços de saúde, e as dificuldades em conseguir atendimento em ambulatórios especializados. Ter como exemplo o ambulatório da Paraíba, que surgiu a partir da demanda dos movimentos sociais e até hoje luta para a regulamentação junto ao Ministério da Saúde, fez perceber os entraves burocráticos que dificultam e protelam a assistência integral a essas pessoas.

No último encontro de discussões do GTTrans, "Violência e preconceito" foi tema de discussão. Com reportagens televisivas veiculadas recentemente, a militante da ASTRAL, Jeany Martins, conseguiu tornar o último encontro um evento de chamada para a luta contra a transfobia. Os participantes, embora tivessem contato desde o primeiro encontro com a temática, nessa reunião puderam discutir com a militante sobre formas de diminuir o preconceito na comunidade e de superá-lo com práticas mais humanizadas.

A finalização do projeto de extensão ao qual o GTTrans estava vinculado se deu com a realização do II Fórum de Saúde Integral da Pessoa Trans. Com tema "A emergência das mudanças sociais e adequação dos serviços de saúde", o evento durou dois dias de palestras, rodas de conversas e debates sobre diversos âmbitos sobre a transexualidade. Por acontecer no Centro de Vivências do campus, o evento teve grande visibilidade. Diversos profissionais e representantes dos movimentos sociais estiveram juntos, discutindo sobre a terapia hormonal para transição de gênero, depressão, processo judicial de mudança de nome e gênero, entre outras discussões que estiveram inclusas no GTTrans, mas que foram reafirmadas e expandidas no fórum.

\section{CONCLUSÃO}

As discussões no GTTrans fazem refletir e compreender a transexualidade como expressão de gênero que, como tal, se relaciona com determinadas demandas na área da saúde.

Espera-se que@s participantes, futur@s profissionais da área da saúde, compreendam as pessoas trans em sua totalidade, contribuindo para que os serviços em que estarão inserid@s não aumentem a discriminação, já tão presente e naturalizada na sociedade.

A universidade, como formadora desses profissionais pode fazer com que os serviços de saúde sejam melhorados, uma vez que o egresso se tornará um construtor de saberes e boas práticas naquele ambiente. Dessa forma, ressalta-se a importância e necessidade de inclusão das políticas trans no ensino e na educação continuada.

\section{REFERÊNCIAS BIBLIOGRÁFICAS}

1. BRASIL. Ministério da Saúde. Portaria n. 2.836/GM, de 01 de dezembro de 2011. Institui, no âmbito do Sistema Único de Saúde (SUS), a Política Nacional de Saúde Integral de Lésbicas, Gays, Bissexuais, Travestis e Transexuais (Política Nacional de Saúde Integral LGBT). 
Diário Oficial da União, Brasília, DF, 02 de dezembro de 2011, 2011.

2. BRASIL. Ministério da Saúde. Portaria n. 1.707/GM, de 18 de agosto de 2008. Institui, no âmbito do SUS, o Processo Transexualizador, a ser implantado nas unidades federadas, respeitadas as competências das três esferas de gestão. Diário Oficial da União, Brasília, DF, 19 de agosto de 2008, 2008a.

3. BRASIL. Ministério da Saúde. Portaria n. 457/SAS, de 19 de agosto de 2008. Regulamenta o Processo Transexualizador no SUS. Diário Oficial da União, Brasília, DF, 20 de agosto de 2008, 2008b.

4. BRASIL. Ministério da Saúde. Portaria n. 2.803/GM, de 19 de novembro de 2013. Redefine e amplia o Processo Transexualizador no Sistema Único de Saúde (SUS). Diário Oficial da União, Brasília, DF, 20 de novembro de 2013, 2013 c.

5. De gravata e unha vermelha [documentário]. Direção: Miriam Chnaiderman, Produção: Reinaldo Pinheiro. Brasil: Sequencia1; 2014.

6. Bento B, Pelúcio, L. Despatologização do gênero: a politização das identidades abjetas. Rev. Estud. Fem., Florianópolis. 2012; 20(2):559-68.

7. Facchini R. Sopa de letrinhas? Movimento homossexual e produção de identidades coletivas nos anos 90 .

Rio de Janeiro: Garamond Seção Garamond Universitária, 2005;6. 304 p.

8. Carvalho M, Carrara S. Em direito a um futuro trans? Contribuição para a história do movimento de travestis e transexuais no Brasil. Sex. Salud Soc. 2013;(14): 319-51.

9. Santos AB, S Helena Eri, Merchan-Hamann E. Processo de formação das representações sociais sobre transexualidade dos profissionais de saúde: possíveis caminhos para superação do preconceito. Ciênc. saúde coletiva. 2014;19(11):4545-54.

10. Santos TZ. Quando o "estranho" resolve se aproximar: a presença da professora transexual e as representações de gênero e sexualidade no ambiente escolar. Tubarão. Dissertação [Mestrado em Educação] - Universidade do Sul de Santa Catarina; 2015.

Artigo apresentado em 28/09/2016

Artigo aprovado em 20/06/2017

Artigo publicado no sistema em 22/09/2017 